\title{
Perception-Based Contrast Enhancement of Images
}

\author{
ADITI MAJUMDER and SANDY IRANI \\ University of California, Irvine
}

\begin{abstract}
Study of contrast sensitivity of the human eye shows that our suprathreshold contrast sensitivity follows the Weber Law and, hence, increases proportionally with the increase in the mean local luminance. In this paper, we effectively apply this fact to design a contrast-enhancement method for images that improves the local image contrast by controlling the local image gradient with a single parameter. Unlike previous methods, we achieve this without explicit segmentation of the image, either in the spatial (multiscale) or frequency (multiresolution) domain. We pose the contrast enhancement as an optimization problem that maximizes the average local contrast of an image strictly constrained by a perceptual constraint derived directly from the Weber Law. We then propose a greedy heuristic, controlled by a single parameter, to approximate this optimization problem.
\end{abstract}

Categories and Subject Descriptors: I.3.3 [Computer Graphics]: Picture/Image Generation-Display algorithms; I.4.0 [Image Processing and Computer Vision]: General-Image displays; I.4.8 [Image Processing and Computer Vision]: Scene Analysis-Color, hotometry

General Terms: Contrast, Color, Perception, Displays

Additional Key Words and Phrases: Human perception, contrast sensitivity, contrast enhancement

ACM Reference Format:

Majumder, A. and Irani, S. 2007. Perception-based contrast enhancement of images. ACM Trans. Appl. Percpt. 4, 3, Article 17 (July 2007), 22 pages. DOI = 10.1145/1278387.1278391 http://doi.acm.org/10.1145/1278387.1278391

\section{INTRODUCTION}

The sensitivity of the human eye to spatially varying contrast is a well-studied problem in the perception literature and has been studied at two levels: threshold and suprathreshold. Threshold contrast sensitivity studies the minimum contrast required for human detection of a pattern, while suprathreshold contrast studies the perceived contrast when it is above the minimum threshold level. These studies show that the contrast sensitivity of humans at suprathreshold levels follows the Weber law and, hence, can be quantified with a single parameter [Valois and Valois 1990]. In this paper, we use this suprathreshold contrast sensitivity to design a new contrast-enhancement technique for $2 \mathrm{D}$ images.

The problem of enhancing contrast of images enjoys much attention and spans a wide gamut of applications, ranging from improving visual quality of photographs acquired with poor illumination [Oakley and Satherley 1998; Rahman et al. 1996] to medical imaging [Boccignone and Picariello 1997]. Common techniques for global contrast enhancements, like global stretching and histogram equalization, do not always produce good results, especially for images with large spatial variation in contrast. To

Authors' address: Aditi Majumder and Sandy Irani, Department of Computer Science, University of California, Irvine, CA 92697. Permission to make digital or hard copies of part or all of this work for personal or classroom use is granted without fee provided that copies are not made or distributed for profit or direct commercial advantage and that copies show this notice on the first page or initial screen of a display along with the full citation. Copyrights for components of this work owned by others than ACM must be honored. Abstracting with credit is permitted. To copy otherwise, to republish, to post on servers, to redistribute to lists, or to use any component of this work in other works requires prior specific permission and/or a fee. Permissions may be requested from Publications Dept., ACM, Inc., 2 Penn Plaza, Suite 701, New York, NY 10121-0701 USA, fax +1 (212) 869-0481, or permissions@acm.org.

(c) 2007 ACM 1544-3558/2007/07-ART17 \$5.00 DOI 10.1145/1278387.1278391 http://doi.acm.org/10.1145/1278387.1278391

ACM Transactions on Applied Perception, Vol. 4, No. 3, Article 17, Publication date: July 2007. 
address this issue, a large number of local contrast enhancement methods have been proposed that use some form of image segmentation either in the spatial(multiscale) or frequency(multiresolution) domain followed by the application of different contrast-enhancement operators on the segments. These approaches differ in the way they generate the multiscale or multiresolution image representation, or in the contrast-enhancement operators they use to enhance contrast after segmentation. Image segmentation has been achieved using methods, such as anisotropic diffusion [Boccignone and Picariello 1997], nonlinear pyramidal techniques [Toet 1992], multiscale morphological techniques [Toet 1990; Mukhopadhyay and Chanda 2002], multiresolution splines [Burt and Adelson 1983], or mountain clustering [Hanmandlu et al. 2001]. Contrast enhancement of the segments has been achieved using morphological operators [Mukhopadhyay and Chanda 2002], wavelet transformations [Velde 1999], curvelet transformations [Stark et al. 2003], k-sigma clipping [Munteanu and Rosa 2001; Rahman et al. 1996], fuzzy logic [Hanmandlu et al. 2000, 2001], genetic algorithms [Shyu and Leou 1998], and operators based on retinex theory [Munteanu and Rosa 2001; Rahman et al. 1996].

In this paper, we present a local contrast-enhancement method driven by an objective function that is controlled by a single parameter derived from the suprathreshold contrast sensitivity of the eye. The perception of contrast is directly related to the local luminance difference, i.e., the local luminance gradient at any point in the image. Our goal then is to enhance these gradients. Methods dealing with gradient manipulation need to integrate the gradient field for image reconstruction. This is an approximately invertible problem, achieved by solving the Poisson equation, and has been used recently to achieve contrast enhancement and seamless image editing [Fattal et al. 2002; Prez et al. 2003]. However, these methods are often cumbersome to implement, because they involve differential equations dealing with millions of variables and complex boundary conditions. Instead, we achieve gradient enhancement by treating images as height fields and processing them in a way that can be controlled by the single parameter derived from the suprathreshold human contrast sensitivity that follows the Weber law. We pose this as an optimization problem that maximizes the local average contrast in an image strictly guided by a perceptual constraint derived directly from the Weber law. In addition, the range of the color values are strictly constrained to avoid artifacts because of saturation of colors. To solve this optimization problem, we propose a new greedy iterative algorithm. We compare the results from this algorithm with existing different global and local contrast-enhancement techniques and show that our results are superior than any traditional or state-of-the art contrast enhancement techniques. By imposing explicit constraints in our optimization formulation, we are able to avoid all common artifacts of contrast enhancement like halos, intensity burn-out, hue shift, and introduction of noise.

\section{WEBER LAW-BASED SUPRATHRESHOLD CONTRAST SENSITIVITY}

In this section, we derive the equation that guides the sensitivity of the human eye to luminance differences at different mean luminance levels. Contrast detection has been studied in vision perception literature for decades [Valois and Valois 1990]. Threshold contrast-sensitivity functions (CSF) define the minimum contrast required to detect a sinusoidal grating of a particular mean luminance and spatial frequency. These are bow-shaped plots with peak sensitivity at about 3 to 5 cycles/degree. Further, the peak sensitivity decreases with the decrease in the mean luminance.

Most of our everyday vision, however, is at suprathreshold (above threshold) levels (i.e., clearly visible) and, hence, above the range of threshold contrast. Recently, there has been a great deal of work to study the contrast discrimination sensitivity of humans for such suprathreshold levels. Of this, we are particularly interested in the study of contrast increments in the context of our contrast-enhancement application. Whittle [1986] presents one of the most comprehensive studies in this direction. This shows that the contrast-threshold function for suprathreshold stimuli is quite flat. This indicates that to generate a perceived change in contrast, $\partial C$, that is beyond the threshold for discrimination, $\partial C$ and 
the local contrast $C$ are related by

$$
\frac{\partial C}{C} \geq \lambda
$$

where $\lambda$ is a constant. Note that the above equation is identical to Weber law. This implies that our suprathreshold contrast sensitivity follows the Weber law. Hence, to achieve visible contrast enhancements, higher luminance patterns need higher contrast increments. This observation forms the mainstay of our contrast-enhancement method.

Equation 1 can be generalized for different spatial frequencies. A recent study [Kingdom and Whittle 1996] showed that the character of contrast discrimination is similar for both sinusoidal and square waves of different spatial frequencies. This finding is corroborated by other work [Barten 1999; Georgeson and Sullivan 1975] confirming that the suprathreshold contrast discrimination characteristics show little variation across spatial frequencies. Also, Peli [1990] and Wilson [1991] has shown the contrast perception to be a quasi local phenomenon, mainly because we use our foveal vision to estimate local contrast.

Using all the above, we derive a simple equation for local contrast enhancement of images. We define the local contrast of an image to be proportional to the local gradient of the image. In other words,

$$
C \propto \frac{\partial I}{\partial x}
$$

where $I(x, y)$ is the image and $C$ is the contrast. As mentioned before, Eq. (1) indicates that to achieve the same perceived increase in contrast across an image, larger gradients have to be stretched more than smaller gradients. In fact, as per Weber law, the stretching should be performed in such a fashion that the contrast increment is proportional to the initial gradient. Thus, the enhanced contrast $C^{\prime}$ is given by

$$
C^{\prime}=\frac{\partial I^{\prime}}{\partial x} \geq(1+\lambda) \frac{\partial I}{\partial x}
$$

where $I^{\prime}(x, y)$ is the contrast-enhanced image. Using the above facts, we express the contrast enhancement of an image $I(x, y)$ by a single parameter $\tau$ as

$$
1 \leq \frac{\frac{\partial I^{\prime}}{\partial x}}{\frac{\partial I}{\partial x}} \leq(1+\tau)
$$

where $\tau \geq \lambda$. The lower bound assures that contrast reduction does not occur at any point in the image and the upper bound assures that the contrast enhancement is bounded and visible. Mantiuk et al. [2006] has shown the constant $\lambda$ to be close to 1 by fitting a curve to the experimental data of Whittle [1986]. Thus contrast enhancement in the images will only be visible for $(1+\tau) \geq 2$, assuring that the Eq. (3) is satisfied. Equation (4), though simple, is very effective in practice to achieve contrast enhancement of images.

\section{THE METHOD FOR GRAY IMAGES}

We pose the local contrast-enhancement problem as an optimization problem. We design a scalar optimization function, derived from Eq. (2), that captures the overall contrast of an image and seeks to maximize it, subject to the constraint described by Eq. (4). In addition, we also constrain the color range of the output image to avoid any artifacts, like burning and dodging, halo, and hue shift. 


\subsection{Optimization Problem}

First, we formulate the contrast-enhancement optimization problem for gray images. We consider the intensity values of a gray image to be representative of the luminance values at the pixel locations and assume a linear image-capture device. Note that the gamma of the image-capture device can be easily reconstructed with existing methods [Debevec and Malik 1997] and used to linearize the images before applying contrast-enhancement techniques. In the worst case of unknown device gamma, a quadratic function can be applied to get a close to linear approximation.

We pose the optimization problem as follows. We propose to maximize the objective function

$$
f(\Omega)=\frac{1}{4|\Omega|} \sum_{p \in \Omega} \sum_{q \in N_{4}(p)} \frac{I^{\prime}(p)-I^{\prime}(q)}{I(p)-I(q)}
$$

subject to a perceptual constraint

$$
1 \leq \frac{I^{\prime}(p)-I^{\prime}(q)}{I(p)-I(q)} \leq(1+\tau)
$$

and a saturation constraint

$$
L \leq I^{\prime}(p) \leq U
$$

where scalar functions $I(p)$ and $I^{\prime}(p)$ represent the gray values at pixel $p$ of the input and output images, respectively, $\Omega$ denotes set of pixels that makes up the image, $|\Omega|$ denotes the cardinality of $\Omega$, $N_{4}(p)$ denotes the set of four neighbors of $p, L$ and $U$ are the lower and upper bounds on the gray values (e.g., $L=0$ and $U=255$ for images that have gray values between 0 and 255), and $\tau>0$ is the single parameter that controls the amount of enhancement achieved. This objective function is derived from Eq. (2) as a sum of the perceived local contrast over the whole image, expressed in the discrete domain. It also acts as a metric to quantify the amount of enhancement achieved. The perceptual constraint (Eq. 6) is derived directly from Eq. (4) by expressing it in the discrete domain. The lower bound in this constraint assures two properties: the gradients are never shrunk and the sign of the gradients are preserved. Finally, the saturation constraint (Eq. 7) ensures that the output image does not have saturated intensity values. Note that the saturation constraint does not control the gradient, but just the range of values a pixel is allowed to have. Thus, the pixels in the very dark or very bright regions of the image will still have their gradients enhanced.

\subsection{Greedy Iterative Algorithm}

We propose an iterative, greedy algorithm to try to maximize the objective function above subject to the constraints. Being local in nature, our method adapts to the changing local contrast across the image, achieving different degrees of enhancement at different spatial locations of the image.

Our algorithm is based on the fundamental observation that given two neighboring pixels with gray values $r$ and $s, r \neq s$, scaling them both by a factor of $(1+\tau)$ results in $r^{\prime}$ and $s^{\prime}$, such that

$$
\frac{r^{\prime}-s^{\prime}}{r-s}=(1+\tau)
$$

Thus, if we simply scale the values $I(p), \forall p \in \Omega$, by a factor of $(1+\tau)$, we obtain the maximum possible value for $f(\Omega)$. However, this could cause violation of Eq. (7) at some pixel $p$, leading to saturation of intensity at that point. To avoid this, we adopt an iterative strategy, employing a greedy approach at each iteration.

We consider the image $I$ as a height field (along the $\mathrm{Z}$ axis) sampled at the grid points of a $m \times n$ uniform grid (on the XY plane). This set of samples represents $\Omega$ for a $m \times n$ rectangular image. Thus, every pixel $p \in \Omega$ is a grid point and the height at $p, I(p)$, is within $L$ and $U$. 
For each iteration, we consider a plane perpendicular to the $\mathrm{Z}$ axis at $b, L \leq b \leq U$. Next, we generate a $m \times n$ matrix $R$ by simple thresholding of $I$, identifying the regions of the height field $I$, which are above the plane $b$ as

$$
R(i, j)=\left\{\begin{array}{l}
1 \text { if } I(i, j)>b \\
0 \text { if } I(i, j) \leq b
\end{array}\right.
$$

Next, we identify the four-connected, nonzero components in $\mathrm{R}$ and label them. Each such component, represented by $h_{i}^{b}$, is called a hillock; the subscript denotes the component number and the superscript denotes the plane used to define the hillocks. Next, each of these hillocks is scaled up by an amount such that no pixel belonging to the hillock is pushed beyond $U$ or has the gradient around it enhanced by a factor of more than $(1+\tau)$.

Our method involves successively sweeping threshold planes $b_{i}$ such that $L \leq b_{i}<U$ and at each sweep, greedily scaling the hillocks respecting the constraints. Note that as we sweep successive planes, a hillock $h_{i}^{b}$ can split into $h_{j}^{b+1}$ and $h_{k}^{b+1}$ or remain unchanged, but two hillocks $h_{s}^{b}$ and $h_{t}^{b}$ can never merge to form $h_{u}^{b+1}$. This results from the fact that our threshold plane strictly increases from one sweep to the next and, hence, the pixels examined at a stage are a subset of the pixels examined at previous stages. Thus, we obtain the new hillocks by only searching among hillocks from the immediately preceding sweep.

For low values of $b$, the size of the hillocks are large. Hence, the enhancement achieved on hillocks might not be close to $(1+\tau)$ because of the increased chances of a peak close to $U$ in each hillock. As $b$ increases, the large connected components are divided so that smaller hillocks can be enhanced more than before.

This step of sweeping planes from $\mathrm{L}$ to $\mathrm{U}$ pronounces only the local hillocks of $I$ and the image thus generated is denoted by $I_{1}$. However, further enhancement can be achieved by also enhancing the local valleys. Thus the second stage of the our method applies the same technique to the complement of $I_{1}$ given by $U-I_{1}(p)$. The image generated from the second stage is denoted by $I_{2}$, which is then complemented again to generate the enhanced output image $I^{\prime}=U-I_{2}(p)$.

\subsection{Performance Improvement}

We perform $U-L$ sweeps to generate each of $I_{1}$ and $I_{2}$. In each sweep we identify connected components in a $m \times n$ matrix. Thus, the time complexity of our algorithm is theoretically $O((U-L) m n))$. However, we perform some optimizations to reduce both the space and time complexity of the method.

We observe that hillocks split at local points of minima or saddle point [Koenderink 1984; Witkin 1983]. Thus, we sweep planes only at specific $b_{i}$ s where the height field attains a local minima or saddle point. This helps us to achieve an improved running time complexity of $O(s m n)$, where $s$ is the number of planes swept (number of local maximas, local minimas, and saddle points in the input image). This idea is illustrated in Figure 1. However, note that this example is constructed to illustrate the method and we have exaggerated the enhancements for better comprehension. In practice, many images have numerous local minima and saddle points. The result is that the threshold usually only increases by one or two values in 8-bit gray images. This results in a process that is more time intensive than necessary. Therefore, we have an additional parameter $\Delta$, which is a lower bound on the amount by which $b$ must increase in consecutive passes. For gray images whose values are in the range from 0 to 255 , a $\Delta$ of 5 or 10 still produces excellent results. This reduces the value of $s$ in the running time to be, at most, $255 / \Delta$. The results of these efficiency improvement optimizations are compared in Figure 2.

We also observe that disjoint hillocks do not interact with each other. Thus, to make our method memory efficient, we process each hillock in a depth first manner before proceeding to the next hillock. 

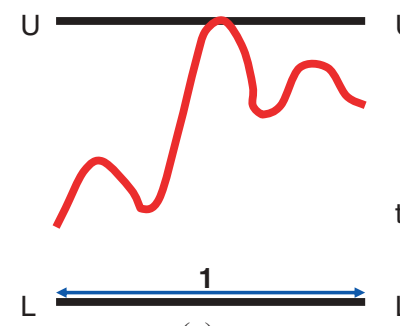

(a)

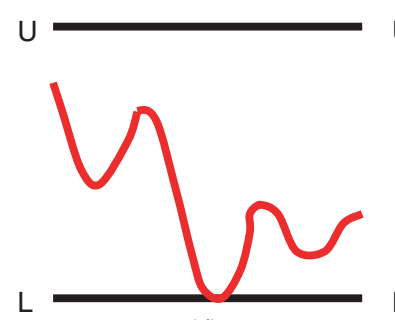

(d)

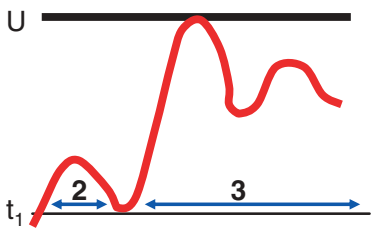

(b)

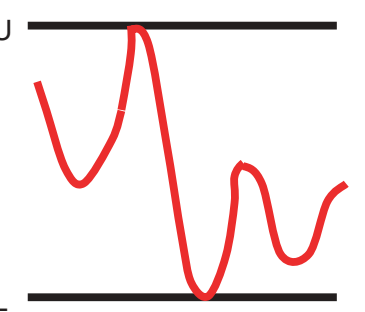

(e)

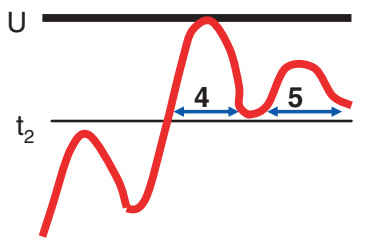

(c)

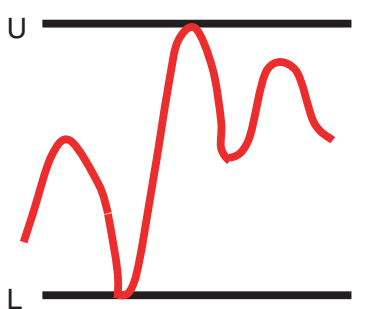

(f)

Fig. 1. Graphs showing some key steps in our algorithm when applied to a 1D signal. In (a), the threshold is zero and there is a single hillock comprised of all the pixels in the input. The hillock is stretched so that the maximum pixel value reaches saturation. In (b), the threshold is increased to $t_{1}$ and hillock 1 is now divided into hillocks 2 and 3 . Hillock 3 can not be stretched any further since its highest pixel is already saturated. However, hillock 2 can be stretched so that the local enhancement of each pixel (as denoted in Eq. 6 ), reaches 1+ $\tau$. Since this is the maximum enhancement that can be achieved, no further processing is performed on hillock 2 . In (c), the threshold is $t_{2}$ and hillock 3 splits into hillock 4 and 5 . Only hillock 5 can be further enhanced, since hillock 4 has a saturated pixel. Hillock 4 is stretched so that the local enhancement of each pixel reaches $1+\tau$. In the second pass, the image from (c) is inverted to produce (d). Hillocks are processed and stretched as in the first pass to produce (e), which is then inverted back to obtain the final enhanced image in (f).

To summarize, following is the pseudocode of our algorithm.

Algorithm Enhance $(\tau, I, L, U)$

Input: $\quad$ Control parameters $\tau$ and $\Delta$

Input Image $I$

Lower and upper bounds $L$ and $U$

Output: Enhanced Image $I^{\prime}$

\section{Begin}

1. $I^{\prime} \leftarrow I$;

2. $I^{\prime}=\operatorname{ProcessHillocks}\left(I^{\prime}, \tau, \Delta\right)$;

3. $I^{\prime} \leftarrow U-I^{\prime}$;

4. $I^{\prime}=\operatorname{ProcessHillocks}\left(I^{\prime}, \tau, \Delta\right)$;

5. $I^{\prime} \leftarrow U-I^{\prime}$;

6. Return $I^{\prime}$;

End

Algorithm ProcessHillocks $(I, \tau, \Delta)$

Input: Input Image $I$

Output: Image $I^{\prime}$

Control parameters $\tau$ and $\Delta$

ACM Transactions on Applied Perception, Vol. 4, No. 3, Article 17, Publication date: July 2007. 


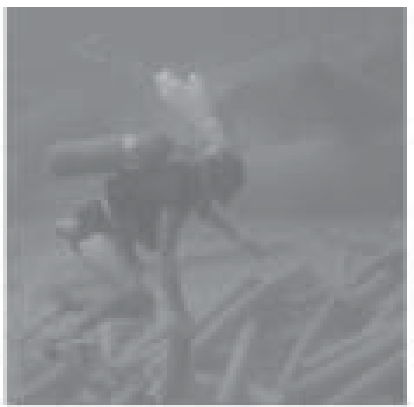

(a)

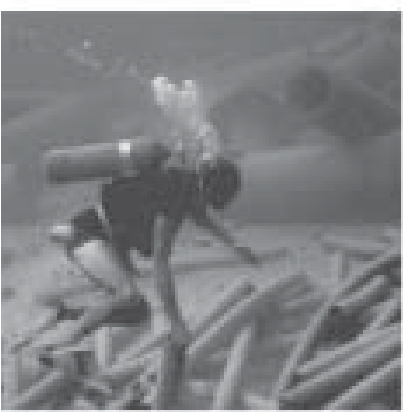

(d)

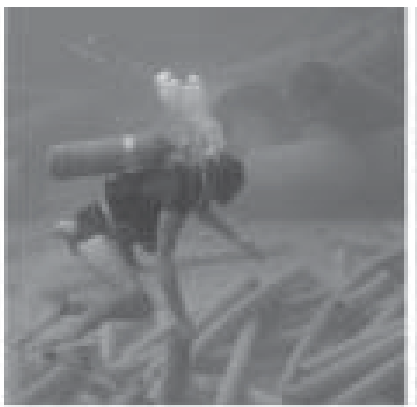

(b)

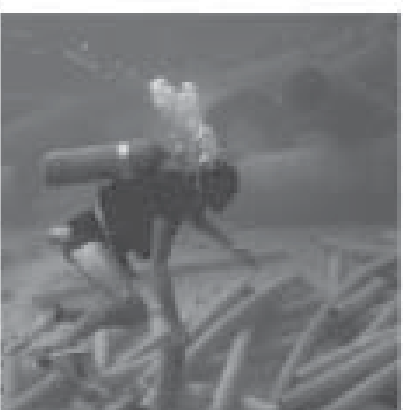

(e)

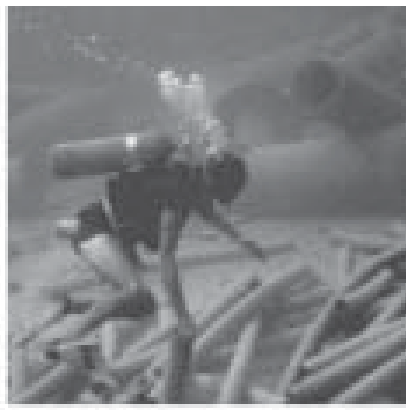

(c)

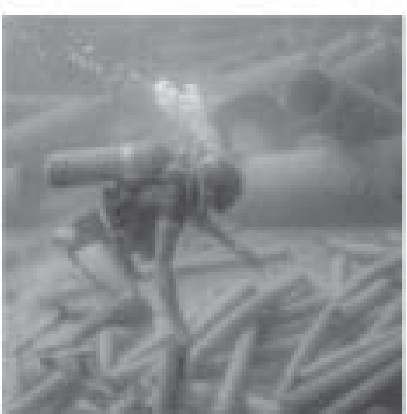

(f)

Fig. 2. The original gray image (a), enhanced gray image using $\tau$ of 0.3 (b), and 2 (c). Note that parts of the image that have achieved saturation for $\tau=0.3$ do not undergo anymore enhancement or show any saturation artifact for higher $\tau$ of 2 . Yet, note further enhancement of areas like the steel band on the oxygen cylinder, the driver's thigh, and the pipes in the background. (c) is generated by sweeping all planes and (d) and (e) are generated by sweeping one of every five and fifty of $(U-L)$ planes, respectively. Note that despite having five times fewer number of sweep planes, (d) is perceptibly indistinguishable from (c). (e) can be distinguished from (c) by the lower contrast background and upper leg of the diver. (f) is the same image enhanced using Fattal's method of stretching gradients using a Poisson solver. Compare this with (c), which is enhanced using our method with $\tau=2$. (f) shows severe noise artifacts. In addition, our method in (c) shows much better performance in driving the darker regions to black, achieving better contrast in the image.

\section{Begin}

1. $I^{\prime}=I$;

2. Create empty stack $S$ of hillocks

3. Create hillock $h$

4. Initialize pixels in $h$ to be all pixels in $I$

5. Initialize threshold(h) to be 0

6. Push $h$ onto $S$

7. While $S$ not empty repeat

8. $\quad \mathrm{h}=\mathrm{S} . \mathrm{pop}()$

9. Find connected components of pixels in $h$ whose value is at least threshold(h)

10. For each connected component $c$

11. Create new hillock $h^{\prime}$

12. Initialize pixels in $h^{\prime}$ to be all pixels in $c$

13. For each pixel $p$ in $h^{\prime}$ 
14. $\quad I^{\prime}(p)=(1+s) *\left(I^{\prime}(p)-t\right)+t$

15. where $t$ is threshold(h) and $s$ is the maximum value over the entire hillock such that none of the constraints are violated.

16. Let threshold $\left(\mathrm{h}^{\prime}\right)$ be the minimum of $I(p)$ over all pixels $p$ in $h^{\prime}$ that are local minima or saddle points and $I(p)$ is at least threshold $\left(\mathrm{h}^{\prime}\right)$

17. Push $h^{\prime}$ onto $S$.

End

Enhance calls the main routine ProcessHillocks on the original image and then on the inverted image so that hillocks get pushed upward and valleys get pushed downward. ProcessHillocks maintains a stack of hillocks. Each hillock maintains a set of pixels, which is disjoint from the pixels in any other hillock. Each hillock also maintains a threshold parameter. In each iteration, the top hillock is popped and the threshold is applied to all the pixels in the hillock. These pixels whose value is above the threshold generate an underlying graph with edges between neighboring pixels. We then compute the connected components of this graph and create a new hillock for each component. In Step 14, all the pixels in each component are then stretched upward as much as possible without violating any of the predefined constraints. Threshold are moved upward and all the resulting hillocks are pushed onto the stack.

\subsection{Results and Performance}

Figure 2 shows the result of applying our method to low-contrast gray images for different values of $\tau$. Note that even after our first optimization of sweeping planes at local minima, maxima and the saddle points, the number of sweep planes can be quite high, i.e., $s$ can be of $O(U-L)$. Hence, for a better performance, we studied the effect of skipping some of the sweep planes and found that we can increase the performance by at least an order of magnitude before seeing visible differences. Figure 3 illustrates this.

Figure 4 compares our method with standard techniques for contrast enhancement that uses global and local histogram equalization, respectively. Figure 2 compares our method on gray images with the recent method proposed in Fattal et al. [2002] that stretches the gradient image directly and then generates the enhanced image from the modified gradient field using a Poisson solver. Figure 5 shows the result of our method on some medical images.

With the ideal parameter of $\Delta=1$, our optimized code takes about $10 \mathrm{~s}$ to process a $500 \times 500$ image. However, by setting $\Delta=10$, we can process the same image in about a couple of seconds. Figure 6 shows the result of our contrast enhancement method on a high-resolution image of 1.5 megapixels with the same $\Delta$, which took around $6 \mathrm{~s}$ to process.

\subsection{Evaluation}

The advantage of our formulation of contrast enhancement as an optimization problem lies in the fact that the objective function, defined in Eq. (5), can be directly used as a metric to evaluate the amount of average contrast enhancement (ACE) achieved across the whole image. Note that according to the constraints of the optimization problem, the maximum average contrast that can be achieved without respecting saturation constraints is given by $1+\tau$. The saturation constraints restrict the actual enhancement achieved to less than or equal to $1+\tau$, while keeping the image free of any artifacts. However, as $\tau$ increases, the effect of the saturation constraint becomes stronger, since larger number of pixels reach saturation when being enhanced and, hence, needs to be restricted not to enhance to 


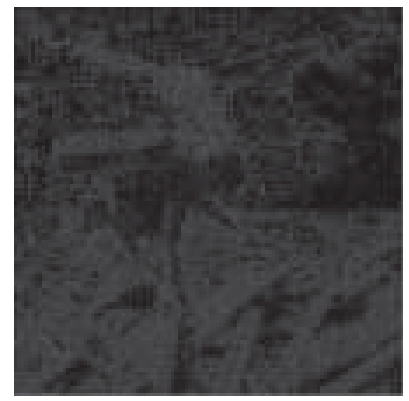

(a)

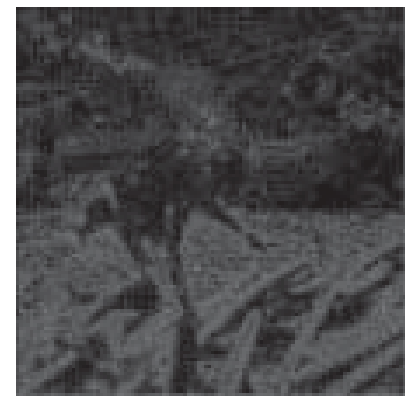

(d)

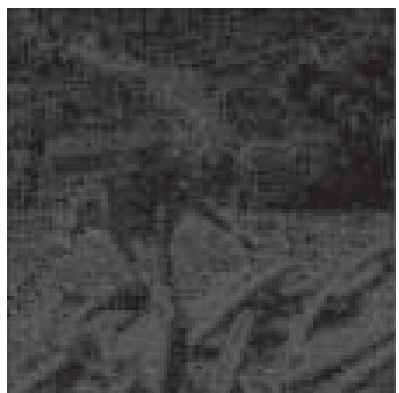

(b)

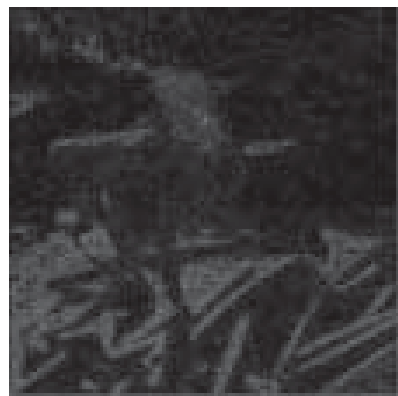

(e)

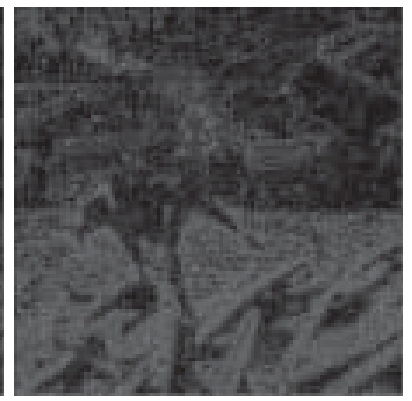

(c)

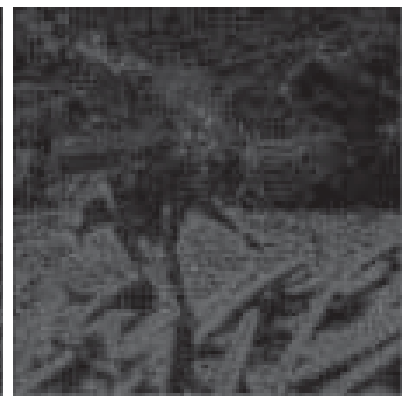

(f)

Fig. 3. Relative local contrast enhancement for the diver image for different parameters on a normalized scale of 0 to 255 , shown as gray scale images. (a) is for $1+\tau=2$, (b) for $1+\tau=3$, and (c) is for $1+\tau=4$. Compare these images and note that as $\tau$ increases, the lower contrast regions of the image shows higher local contrast enhancements. All of (a), (b), and (c) are results of sweeping planes at the intervals of 10 intensity levels. (d) and (e), respectively, show the image corresponding to sweeping planes at every intensity level and at intervals of 50 intensity levels for $1+\tau=4$. Compare these with (c). Note that the contrast enhancement is most widespread for (d), where a plane is swept through every intensity level. Also note the considerable black regions of (e), which shows that contrast enhancement has not occurred in many pixels because of the large interval of the sweeping planes. (f) is the image corresponding to performing only the first pass for $1+\tau=4$ and sweeping planes or intervals of 10 intensity levels. Compare this with (c). Note that the difference is hardly noticeable, which indicates that the most important enhancements are achieved in the first pass itself.

their fullest extent. Hence, with the increase in $\tau$, the actual average enhancement achieved falls more and more away from $1+\tau$. Table I illustrates the ratio of the actual average enhancement achieved and $1+\tau$ for different images for different values of $\tau$. Note that as $(1+\tau)$ increases, though the ACE value increases in an absolute sense, the ratio of $\mathrm{ACE}$ and $(1+\tau)$ decreases, as expected.

Table I also shows that this same metric can be used to evaluate the effect of different optimizations (like skipping some of the sweeping planes and the reverse pass) on the ACE achieved. Note that increasing the number of sweeping planes skipped, indeed decreases the ACE. In addition, this provides us interesting insights in the algorithm. For example, it shows that most of the enhancement is achieved in the first pass itself. The enhancement in the second pass is mostly of details, so skipping the second pass may help us in improving the performance of the method.

In fact, a better metric in this respect is the local contrast enhancement at every pixel of the image. This can give us information on how the contrast has been enhanced in different parts of the image. To illustrate this in Figure 3, we show the images where the intensity at any pixel shows the relative local contrast achieved at that pixel in a normalized scale of 0 to 255 . 


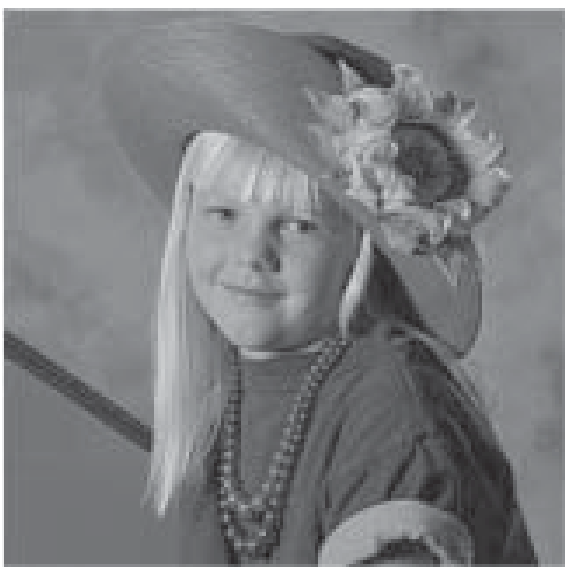

(a)

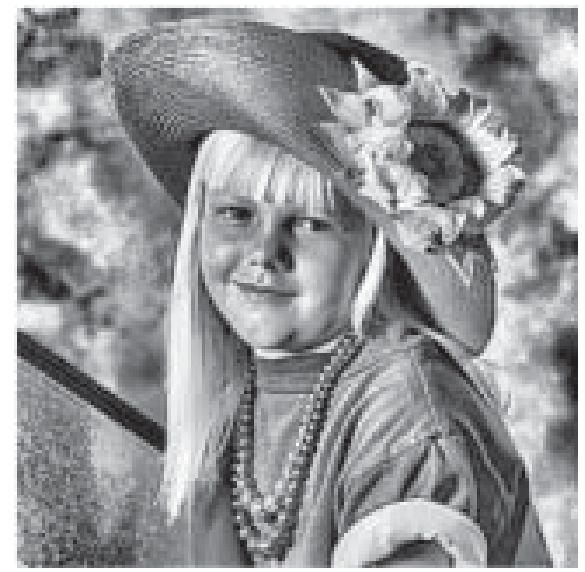

(c)

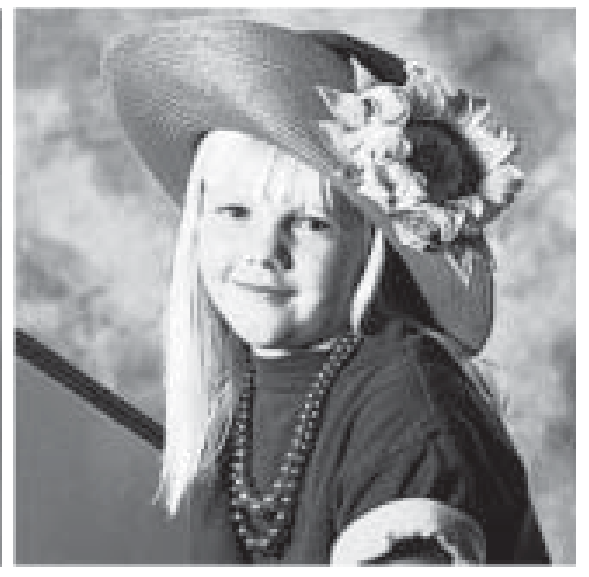

(b)

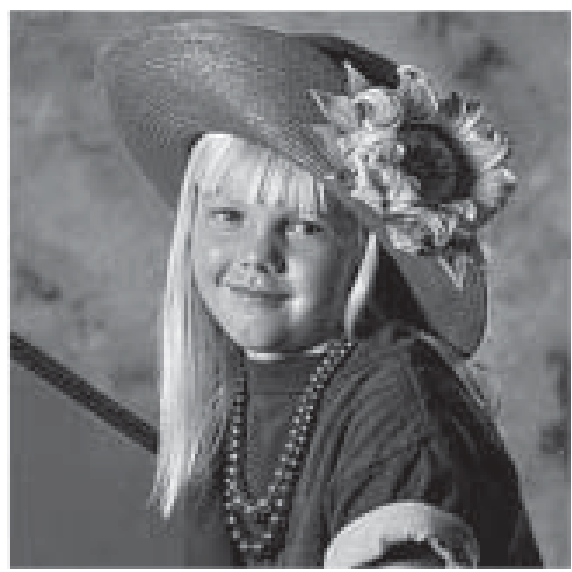

(d)

Fig. 4. The original gray image (a), enhanced using global histogram equalization (b), enhanced using local histogram equalization (c), and enhanced using our method with $\tau=1(\mathrm{~d})$. Note that global histogram equalization leads to oversaturation of parts of the image in (b). While local histogram equalization alleviates that problem, it ends up introducing noise in the background and changes the appearance of parts of the image in (c), like the shirt. Our method in (d) does not suffer from both of these and achieves an image that is closer to the original in its appearance.

\section{EXTENDING TO COLOR IMAGES}

The most obvious way to extend the algorithm presented in the preceding section to color images is to apply the method independently to three different color channels. The results of this is illustrated in Figure 7, this does not assure hue preservation and results in hue-shift artifacts for higher values of $\tau$. This happens when in some pixels of the image, one of the channels saturates while other channels have significant room for enhancement as illustrated in Figure 8.

To avoid this problem, we take the standard approach of separating the luminance and the chrominance channels of the image and then applying our method only on the luminance channel. For this, we first linearly transform RGB values to CIE XYZ space. The XYZ values of the maximum intensity of the primaries are defined by three vectors in the XYZ space, $\bar{R}=\left(X_{R}, Y_{R}, Z_{R}\right), \bar{G}=\left(X_{G}, Y_{G}, Z_{G}\right)$ 


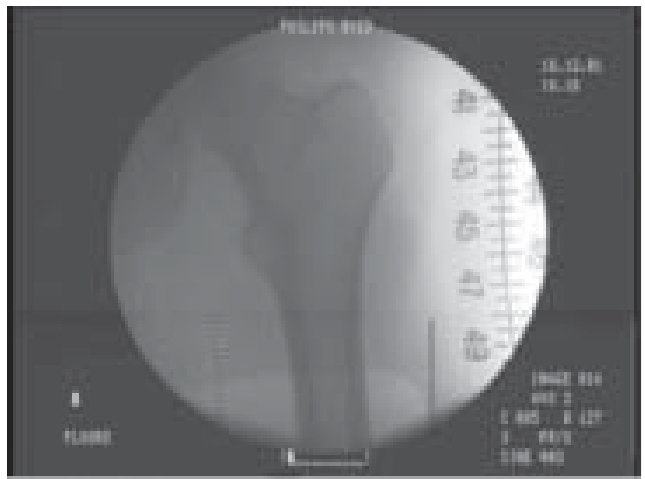

(a)

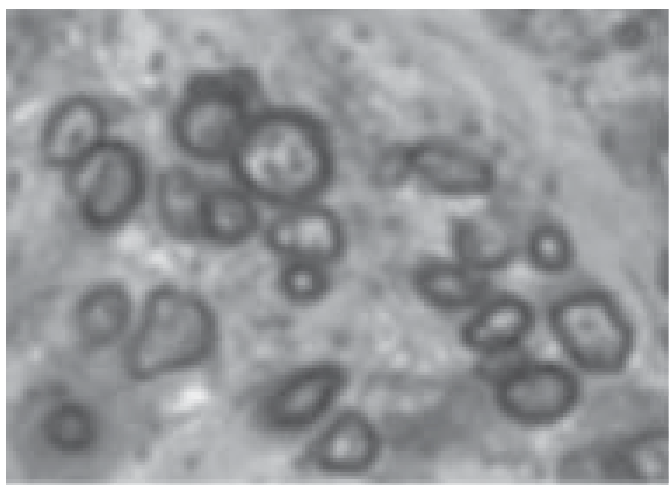

(c)

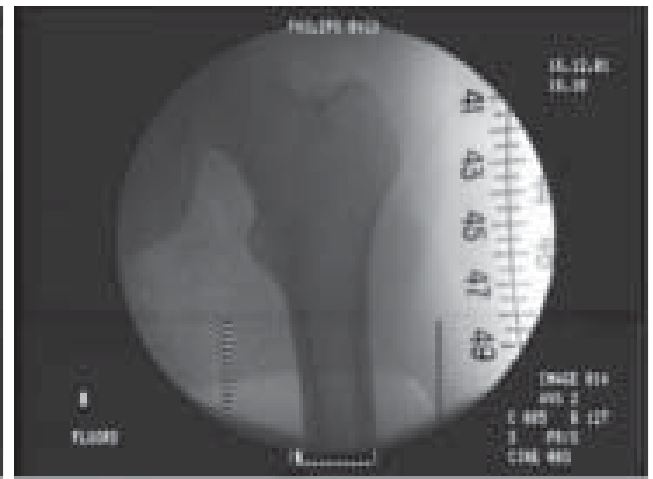

(b)

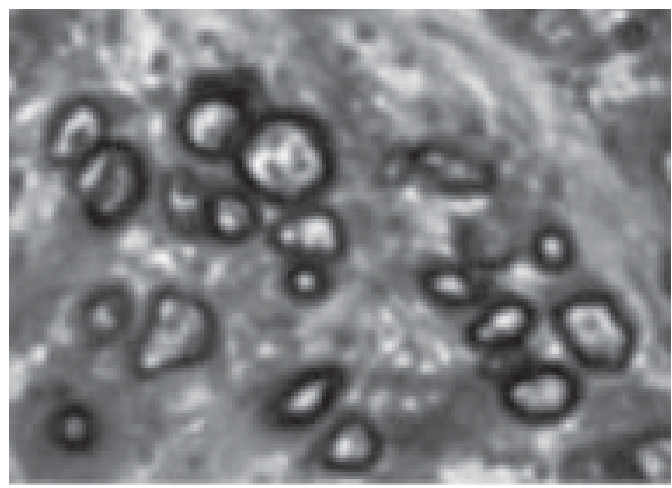

(d)

Fig. 5. The result of our method on some medical images. An x-ray image (a) enhanced with $\tau=1$ (b), and the image of some stem cells taken through a microscope (c) enhanced using $\tau=2(\mathrm{~d})$.

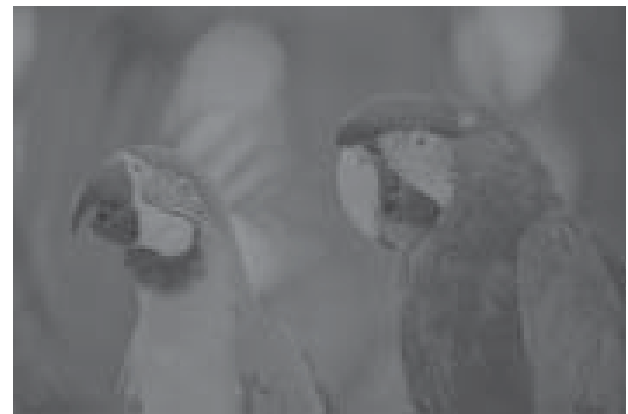

(a)

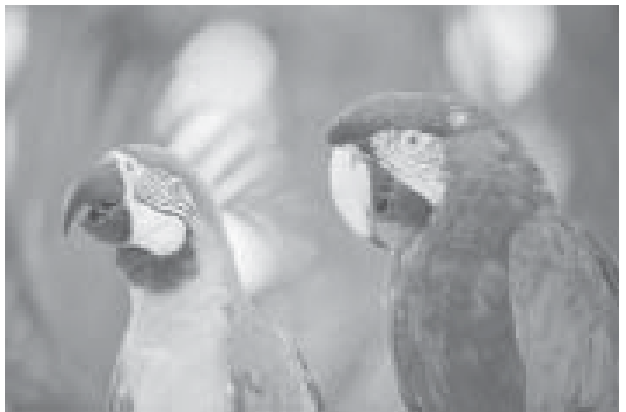

(b)

Fig. 6. (a) The original high-resolution image; (b) enhanced image using our method with $\tau=2.2$ and $\Delta=10$.

and $\bar{B}=\left(X_{B}, Y_{B}, Z_{B}\right)$. These three vectors, in turn, define the color gamut of the RGB device. Any color in the XYZ space that can be expressed as a convex combination of $\bar{R}, \bar{G}$, and $\bar{B}$ is within the device's color gamut and the transformation from RGB to XYZ space is defined by a $3 \times 3$ matrix, whose rows correspond to $\bar{R}, \bar{G}$, and $\bar{B}$. From the converted XYZ values, we obtain the luminance, $Y$, and 
Table I. ACE Achieved by Changing Different Parameters on which the Performance of Our Greedy Algorithm Depends ${ }^{a}$

\begin{tabular}{|l|c|c|c|c|l|}
\hline Image & $1+\tau$ & Step size & Number of Passes & ACE & $\frac{A C E}{1+\tau}$ \\
\hline Diver & 2 & 10 & 2 & 1.43 & 0.715 \\
& 3 & 10 & 2 & 1.80 & 0.6 \\
& 4 & 10 & 2 & 2.18 & 0.545 \\
& 4 & 1 & 2 & 2.39 & 0.598 \\
& 4 & 50 & 2 & 1.32 & 0.33 \\
& 4 & 10 & 1 & 2.13 & 0.532 \\
\hline Blonde & 3 & 10 & 2 & 1.45 & 0.483 \\
& 5 & 10 & 2 & 1.84 & 0.368 \\
\hline
\end{tabular}

${ }^{a}(1+\tau)$ is the parameter controlling the contrast enhancement. Step size defines the gap between the planes which are swept to intersect the luminance field. The number of passes is 2 when both the forward and inverse passes are applied and 1 when only the forward pass is applied.

chromaticity coordinates $(x, y)$ [Giorgianni and Madden 1998] by

$$
x=\frac{X}{X+Y+Z} ; \quad y=\frac{Y}{X+Y+Z}
$$

Note that the change in the chromaticity coordinates $(x, y)$ indicate a change in the chroma (hue and saturation) of the color, but not in luminance. Thus, we perform the contrast enhancement only on luminance $Y$, keeping the chromaticity coordinates, $x$ and $y$, unchanged. Finally, we convert the Yxy image back to the RGB representation.

However, in this case, the saturation constraint can no longer be expressed by a single linear inequality as in Eq. (7). Now, as we enhance $Y$, we have to assure that the enhanced color lies within the RGB gamut, i.e., within the parallelopiped defined by the convex combination of $\bar{R}, \bar{G}$, and $\bar{B}$.

Thus, the luminance enhancement problem can be formulated in a similar fashion as gray-scale images. The color at pixel $p$ given by $C(p)=(X, Y, Z)$ is to be enhanced to $C^{\prime}=\left(X^{\prime}, Y^{\prime}, Z^{\prime}\right)$. The goal is to enhance the luminance $Y$ to $Y^{\prime}$ such that the objective function

$$
f(\Omega)=\frac{1}{4|\Omega|} \sum_{p \in \Omega} \sum_{q \in N_{4}(p)} \frac{Y^{\prime}(p)-Y^{\prime}(q)}{Y(p)-Y(q)}
$$

is maximized subject to a perceptual constraint

$$
1 \leq \frac{Y^{\prime}(p)-Y^{\prime}(q)}{Y(p)-Y(q)} \leq(1+\tau)
$$

and a saturation constraint

$$
\left(X^{\prime}, Y^{\prime}, Z^{\prime}\right)=c_{R} \bar{R}+c_{G} \bar{G}+c_{B} \bar{B}, \quad 0.0 \leq c_{R}, c_{G}, c_{B} \leq 1.0,
$$

Note that Eq. (11) assures that the chromaticity coordinates are not changed and, hence, the chroma is preserved. This optimization is implemented by applying the same technique, explained in Section 3, on the luminance image and by changing the check for saturation constraint as per Eq. (12) and results in hue-preserving contrast enhancement that does not show any hue-shift artifacts. This is illustrated in Figure 8.

The saturation constraint in Eq. (12) implies that the saturation luminance will change from pixel to pixel and is dependent on the chromaticity coordinates at that pixel. This is because of the nonconstant boundary of the 3D color gamut with luminance Y. Hence, Eq. (12) can be imposed by preprocessing the image and deriving a spatially varying luminance saturation envelope whose value on each pixel 


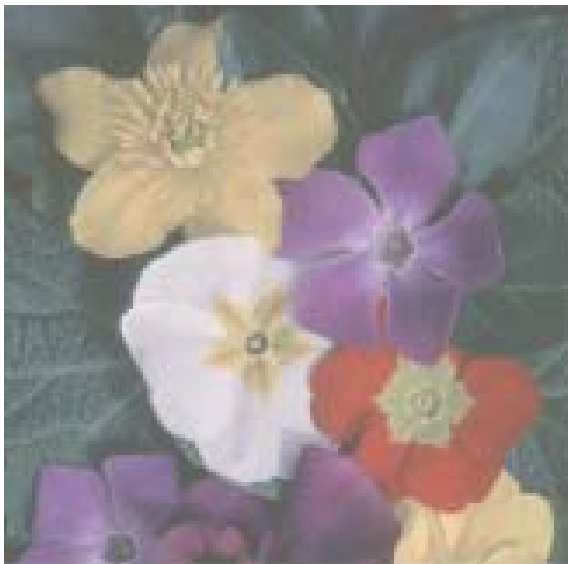

(a)

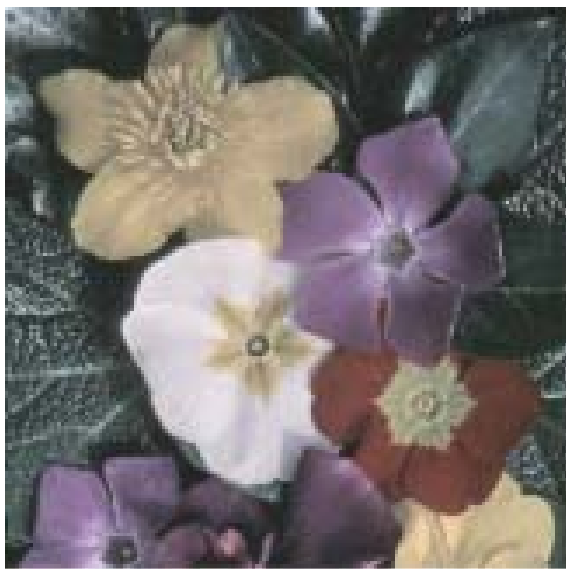

(c)

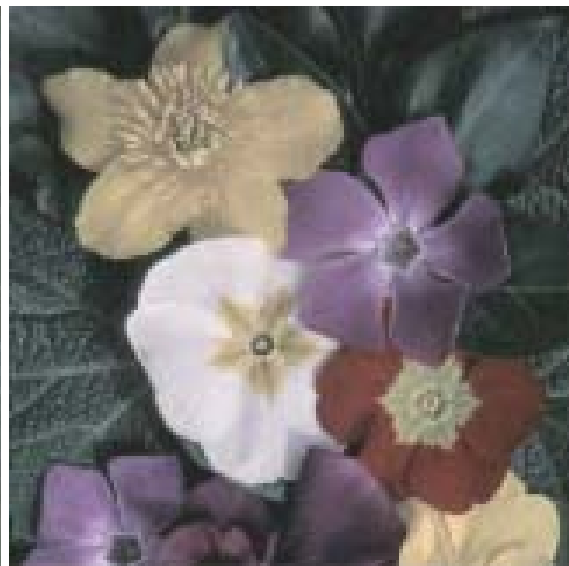

(b)

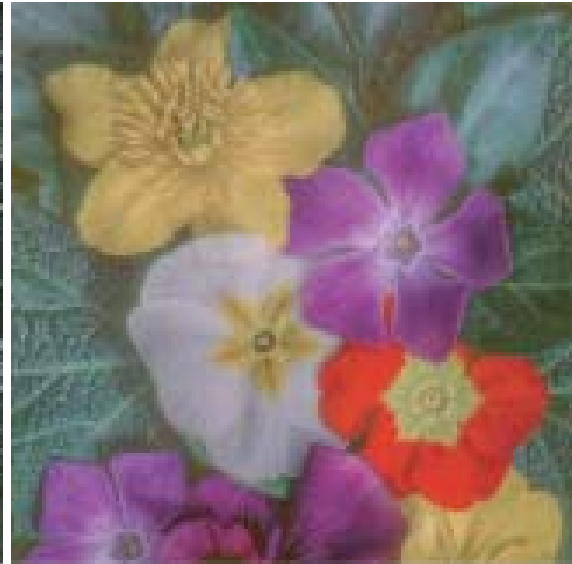

(d)

Fig. 7. Our method applied to the red, green, and blue channel of a color image - the original image (a), enhanced image using $\tau$ of 1 (b), and 9 (c) -note the differences in the yellow regions of the white flower and the venation on the leaves are further enhanced. The same image enhanced by applying Fattal's method is shown in (d). When comparing with the results of our method in (b) and (c), note the haloing artifacts around the white flower and the distinct shift in hue, especially in the red flower, that changes its appearance. It almost appears that (d) is achieved by hue enhancement of (a), rather than a contrast enhancement of (a).

depends on the chroma at that pixel. In this case, during the second pass, when we invert the image with respect to a spatially varying saturation envelope, the relative magnitudes of neighboring pixels may not be maintained. This can lead to switching of directions of luminance gradient at some pixels, which is undesirable. From our study using the evaluation metric in Section 3.5, we found that second pass leads to insignificant change in ACE. Thus, for color images, we only apply the first pass. Further, in case of color images, the luminance $\mathrm{Y}$ is no longer an integer. Hence, $\Delta$ has to be a small floating-point number. We use $\Delta=0.2$ in most of the results shown in this paper.

\subsection{Results}

The most recent contrast enhancement technique is the one developed by Fattal et al. [2002] that does a direct gradient stretching and applies a Poisson solver to get the image back from its gradient field. We 


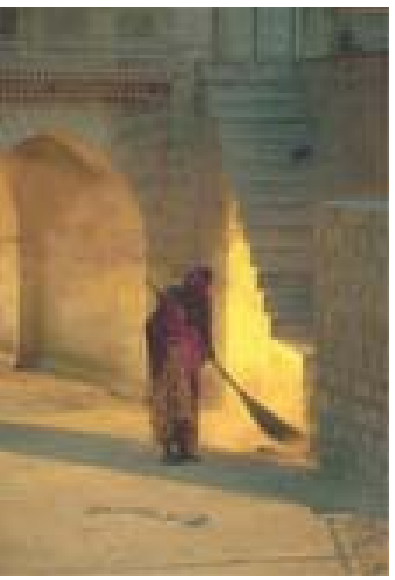

(a)

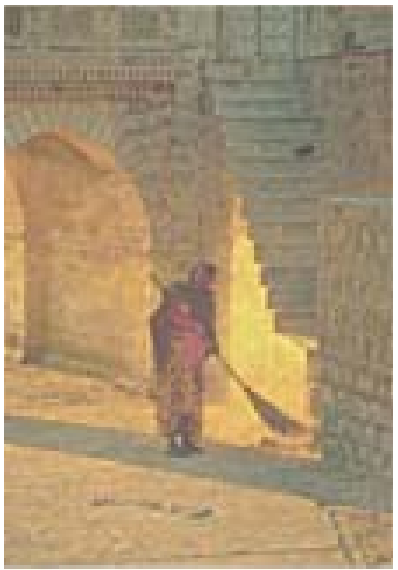

(d)

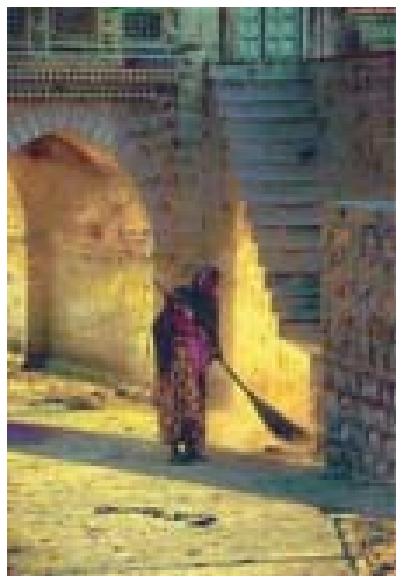

(b)

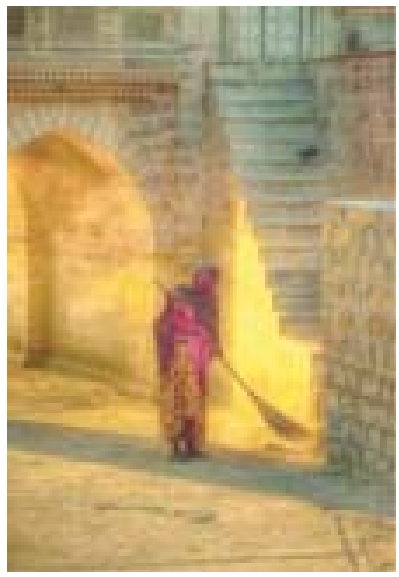

(e)

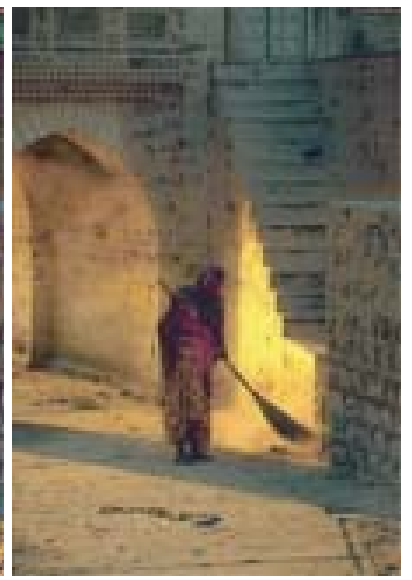

(c)

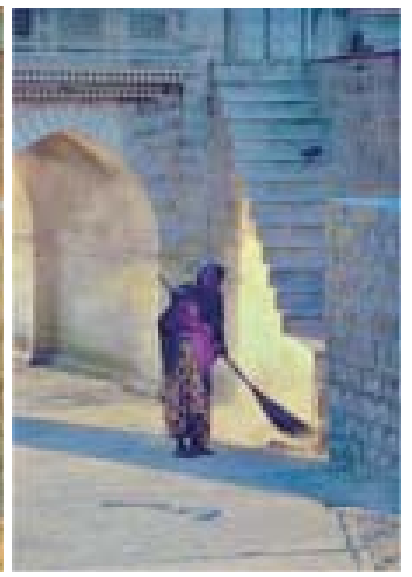

(f)

Fig. 8. The original image (a) and result of our method applied with $\tau=2$ on each of red, green, and blue channels independently (b). Note the severe hue shift toward purple in the stairs, arch, and wall, and toward green on the ledge above the stairs. (c) is the result of first converting the image to luminance and chrominance channels and then applying our method to the luminance channel only. Note that the hue is now preserved. Compare (c) with results of methods based on curvelet transformation [Stark et al. 2003] in (d), manipulation of gradient field inverted back using Poisson solver [Fattal et al. 2002] in (e), and on multiscale retinex theory [Rahman et al. 1996] in (f). Note that (d) and (e) lead to a noisy image, while (e) and (f) significantly change the hue of the image.

compare results from our method with this work in Figures 7 and 8 . Note that we have entirely avoided different kinds of artifacts like halo, noise, and, in particular, hue shift. Figures 8 and 9 compare the result of our method with some existing global and local contrast-enhancement techniques.

Preserving hue is often critical in many applications, like medical and scientific visualization. Our method is especially suited for such applications. To illustrate this, we compared the mean hue shift that occurred when contrast in enhanced using different methods. We first convert the original and the enhanced image to perceptually uniform CIELAB color space. In this color space, the visual difference between two colors is proportional to the Euclidian distance between them. Since the luminance variation between the original and the enhanced image is attributed to the contrast-enhancement procedure, 


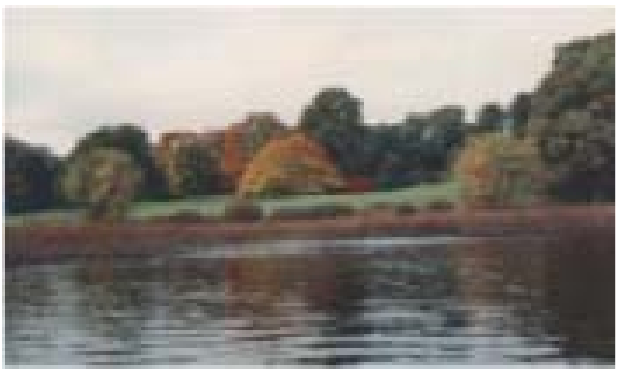

(a)

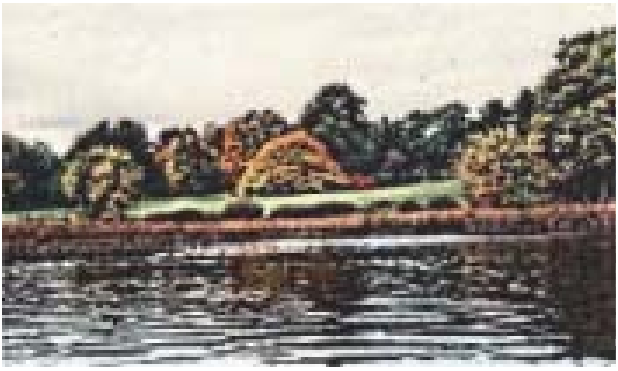

(c)

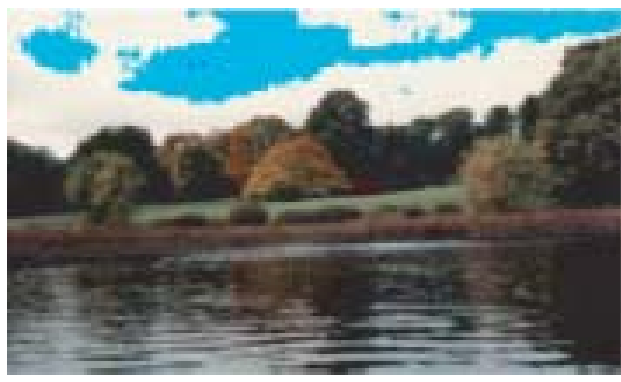

(e)

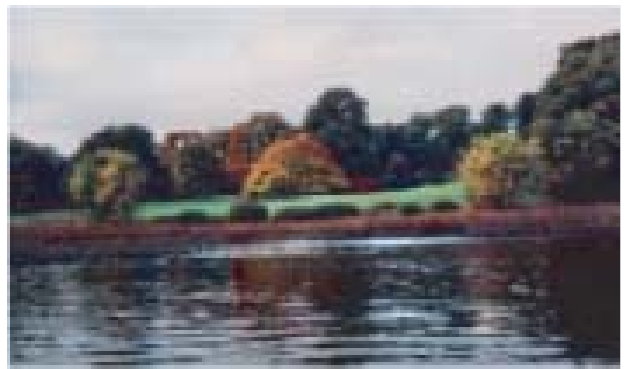

(b)

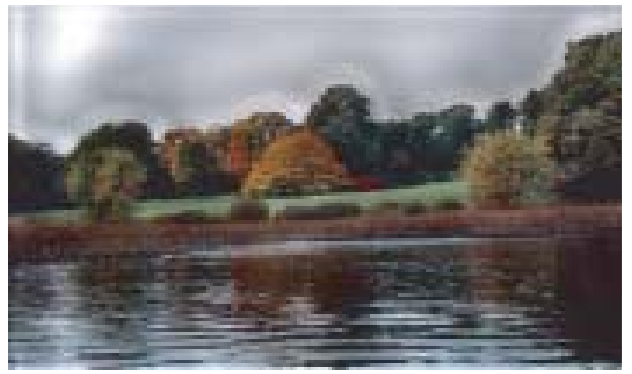

(d)

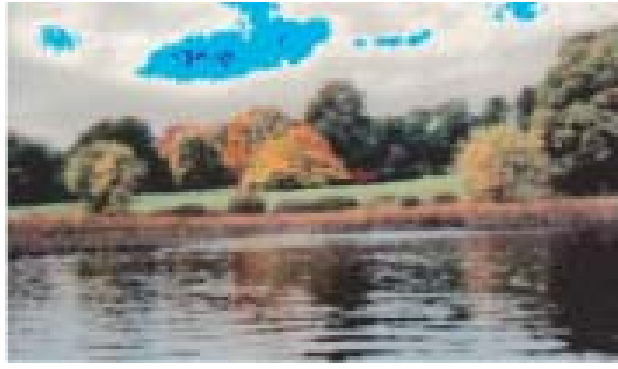

(f)

Fig. 9. (a) The original image; (b) our method with $\tau=2$; (c) multiscale morphology method [Mukhopadhyay and Chanda 2002]. Note the saturation artifacts that gives the image an unrealistic look. (d) Toet's method of multiscale nonlinear pyramid recombination [Toet 1992]. Note the halo artifacts at regions of large change in gradients. (e) Global contrast stretching; (f) global histogram equalization. Both (e) and (f) suffer from saturation artifacts and color blotches.

we find the Euclidian distance between the original and enhanced $\left(a^{*}, b^{*}\right)$ at every pixel. This distance at every pixel is then averaged across all the pixels in the image to calculate the mean hue shift. Table II shows the results. Our RGB to CIELAB color space conversion is performed with reference to a D65 illuminant and a two-degree observer. Note that our method consistently shows the least hue shift when compared with other existing methods.

\subsection{Evaluation and Comparison}

Table III also shows the ACE achieved for different color images for different parameters. Note that ACE can also be used to compare different methods by comparing the ACE achieved by different methods applied to the same images. This is illustrated in Table IV. However this metric cannot penalize the contrast enhanced as a result of saturation/halo artifacts. Hence, note that in Table IV, methods like 
Table II. Comparison of Hue Shift Artifact of Different Methods by Measuring the Average Euclidian Distance between the CIELAB $a^{*}$ and $b^{*}$ Values of the Original and Enhanced Images

\begin{tabular}{|c|c|c|}
\hline Image & Method & Mean Hue Shift \\
\hline \multirow{6}{*}{$\begin{array}{l}\text { River } \\
\text { (Figure 9) }\end{array}$} & Contrast stretching & 6.86 \\
\hline & Global Histogram & 4.67 \\
\hline & Multiscale morphology & 5.69 \\
\hline & Toet & 5.27 \\
\hline & Fattal & 2.60 \\
\hline & Greedy $(1+\tau=3)$ & 2.26 \\
\hline \multirow{2}{*}{$\begin{array}{l}\text { Flower } \\
\text { (Figure 7) }\end{array}$} & Fattal & 17.30 \\
\hline & Greedy $(1+\tau=2)$ & 2.07 \\
\hline \multirow{4}{*}{$\begin{array}{l}\text { Indian Woman } \\
\text { (Figure 8) }\end{array}$} & Fattal & 8.66 \\
\hline & Curvelet & 4.01 \\
\hline & Retinex & 27.02 \\
\hline & Greedy $(1+\tau=3)$ & 2.04 \\
\hline \multirow[t]{4}{*}{ Castle (Figure 10) } & Multiscale retinex with & \\
\hline & color restoration & 12.87 \\
\hline & Greedy $(1+\tau=2)$ & 5.95 \\
\hline & $\begin{array}{l}\text { Greedy }(1+\tau=2) \text { with } \\
\text { tone mapping }\end{array}$ & 10.0 \\
\hline \multirow[t]{4}{*}{ Baby (Figure 11) } & Multiscale retinex with & \\
\hline & color restoration & 4.76 \\
\hline & Greedy $(1+\tau=2)$ & 2.35 \\
\hline & $\begin{array}{l}\text { Greedy }(1+\tau=2) \text { with } \\
\text { tone mapping }\end{array}$ & 4.67 \\
\hline
\end{tabular}

Table III. Comparison of ACE Achieved by Changing Different Parameters on which the Performance of Our Greedy Algorithm Depends ${ }^{a}$

\begin{tabular}{|l|c|c|c|c|}
\hline Image & $1+\tau$ & Step Size & ACE & $\frac{A C E}{1+\tau}$ \\
\hline Flower & 2 & 0.2 & 1.50 & 0.75 \\
& 10 & 0.2 & 2.00 & 0.20 \\
& 4 & 0.2 & 1.73 & 0.43 \\
& 4 & 0.02 & 1.75 & 0.44 \\
\hline Indian woman & 2 & 0.2 & 1.32 & 0.66 \\
& 4 & 0.2 & 1.49 & 0.37 \\
& 6 & 0.2 & 1.59 & 0.27 \\
& 10 & 0.2 & 1.68 & 0.17 \\
\hline
\end{tabular}

${ }^{a}(1+\tau)$ is the parameter controlling the contrast enhancement. Step size defines the gap between the planes, which are swept to intersect the luminance field.

contrast stretching, global histogram, and multiscale morphology show a high ACE mainly because of their saturation artifacts which, in reality, are not the strength of these methods, but rather a serious shortcoming. Thus, a higher ACE does not always correspond to a better method. We propose ACE as a tool for quantitative analysis of contrast-enhancement methods that would help us study and compare methods and images and provide us with interesting insights. Thus, we do not propose that this metric would be used for automated evaluation of methods that would replace human judgment.

In addition, this metric is not independent of the way the luminance is calculated while applying the method. For example, Fattal's and retinex theory method applies a different transformation than us to separate the luminance and the color channels. We use a different metric to do so while evaluating the 
Table IV. Comparison of the ACE Achieved by Applying Different Methods to the Same Image

\begin{tabular}{|l|l|l|}
\hline Image & Method & ACE \\
\hline Blonde (Figure 4) & Global histogram & 2.29 \\
& Local histogram & 5.77 \\
& Greedy $(1+\tau=3)$ & 1.45 \\
\hline River (Figure 9) & Contrast stretching & 2.85 \\
& Global histogram & 4.11 \\
& Multiscale morphology & 9.55 \\
& Toet & 3.76 \\
& Fattal & 1.93 \\
& Greedy $(1+\tau=3)$ & 1.33 \\
\hline Diver (Figure 2) & Fattal & 2.04 \\
& Greedy $(1+\tau=4)$ & 2.38 \\
\hline Flower (Figure 7) & Fattal & 1.68 \\
& Greedy $(1+\tau=10)$ & 2.00 \\
\hline Indian woman (Figure 8) & Fattal & 1.87 \\
& Curvelet & 2.62 \\
& Retinex & 2.40 \\
& Greedy $(1+\tau=4)$ & 1.49 \\
\hline
\end{tabular}

Table V. Comparison of Perception of Contrast Enhancement by Different Methods Using ACE Formula on CIELAB of Original and Enhanced

Images

\begin{tabular}{|l|l|c|c|}
\hline Image & Method & Artifacts & $\begin{array}{c}\text { ACE on L } \\
\text { of CIELAB }\end{array}$ \\
\hline River (Figure 9) & Contrast stretching & Saturation & 2.34 \\
& Global histogram & Saturation & 2.72 \\
& Multiscale morphology & Halo & 8.99 \\
& Tote & Halo & 3.67 \\
& Fattal & None & 1.09 \\
& Greedy $(1+\tau=2)$ & None & 1.70 \\
\hline Flower (Figure 7) & Fattal & None & 1.84 \\
& Greedy $(1+\tau=4)$ & None & 2.15 \\
\hline Indian woman (Figure 8) & Fattal & None & 1.01 \\
& Curvelet & None & 1.28 \\
& Multiscale retinex & Hue shift & 1.51 \\
& Greedy $(1+\tau=6)$ & None & 1.57 \\
\hline
\end{tabular}

image. Hence, though we perceive a comparable contrast enhancement, the ACE computed does not confirm to our visual evaluation making these values inconsistent.

Thus, for a better perceptual analysis, we used the same ACE metric, but on the $L$ values resulting from converting the original and enhanced images in the CIELAB space. We consider a two-degree observer and a D65 illuminant for this conversion. When we apply the ACE metric to evaluate the contrast enhancement in the CIELAB space, the metric provides us a perceptual measure of the contrast enhancement achieved using different methods. Though this also does not penalize the saturation/halo artifacts, for methods that do not result in serious artifacts, this does provide a metric for the perceived contrast enhancement, as shown in Table V.

In retrospective, our greedy algorithm may seem close to the class of image-enhancement algorithms based on retinex theory and, hence, needs a detailed comparison with these methods. The fundamental retinex theory proposed by Land considers an image to be a pixel-by-pixel multiplication of the ambient illumination and object reflectance [Land 1964; Land and McCann 1971]. Any contrast-enhancement 


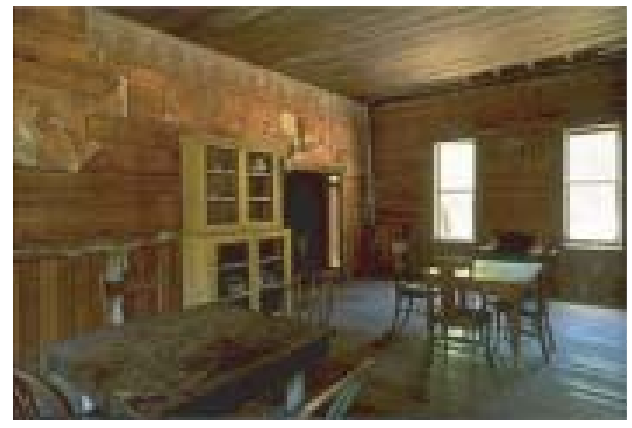

(a)

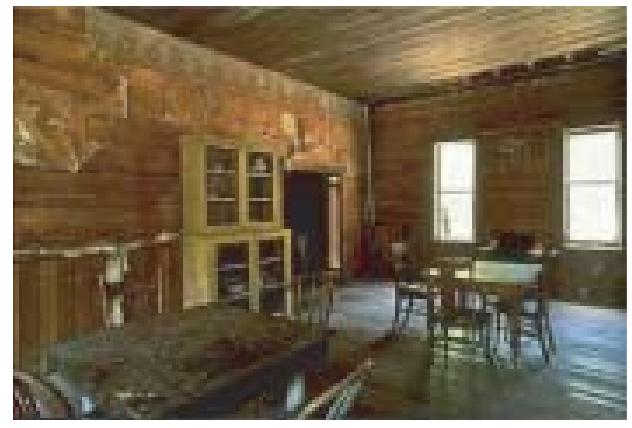

(c)

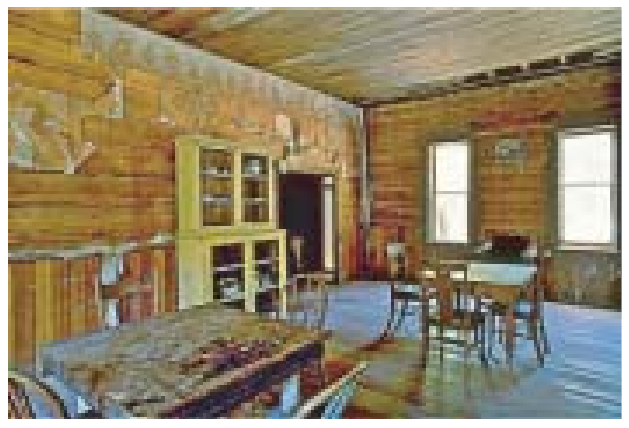

(b)

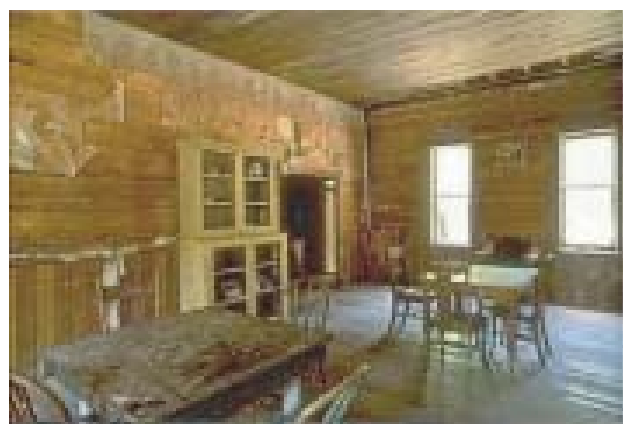

(d)

Fig. 10. (a) The original image; (b) enhanced image using multiscale retinex theory with color restroration; (c) enhanced image using our method with $\tau=1$ and $\Delta=0.2$; and (d) same enhanced image as (c) after tone-mapping using a simple polynomial tonemapping operator. Note that even a simple operator can yield comparable results. Also, note that (b) involves color restoration to account for the severe hue shift resulting from retinex-based methods.

method that uses retinex theory is based on recovering the reflectance function from the image by removing the illumination effects. Since illumination changes are usually smooth, they can be estimated at a lower resolution. Based on this fact, most retinex methods generate a low-resolution illumination image by convolving multiple local pixels using a filter that can be as simple as a box filter. The original image is then divided by this illumination image to expose only the reflectance component of the image. Hence, areas with low illumination will be enhanced more (because of division with a lower number) than those with high illumination. For multiscale methods [Munteanu and Rosa 2001; Rahman et al. 1996], the same technique is applied at different spatial scales to generate the illumination image and then the resultant multiscale reflectance images are combined by some weighting function. However, note that such removal of illumination effects can also be achieved by tone-mapping methods. For example, the most adverse effects of illumination burning or dodging details are usually noticed in high dynamic-range imaging. Such effects can be removed to bring out details using sophisticated tonemapping operators [Reinhard et al. 2002, 2005]. Thus, what such retinex theory-based methods achieve are both tone reproduction and contrast enhancement. In fact, probably for the same reason, they are usually referred to as image-enhancement rather than contrast-enhancement methods.

The goal of contrast enhancement however is different from tone mapping. Arguably, for aesthetic reasons, it is not desirable to remove the effects of illumination. This is where our method differs, in principle, from the retinex based methods. Our method concentrates only on contrast enhancement 


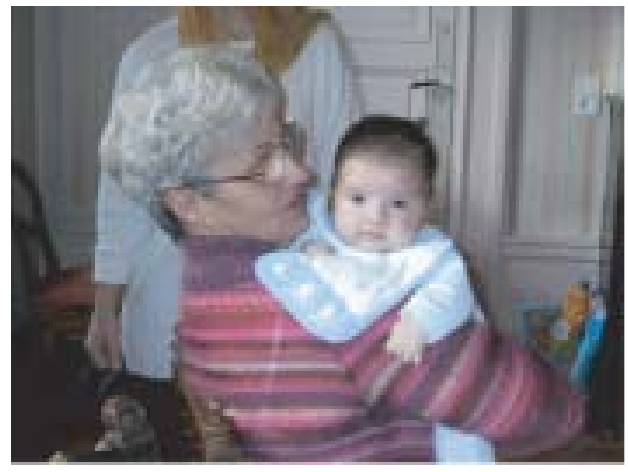

(a)

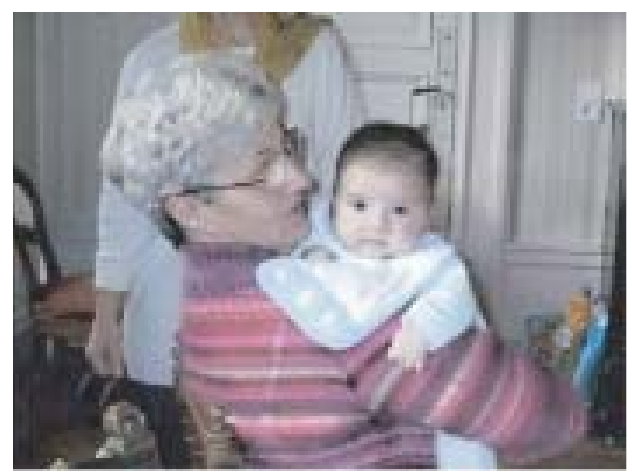

(c)

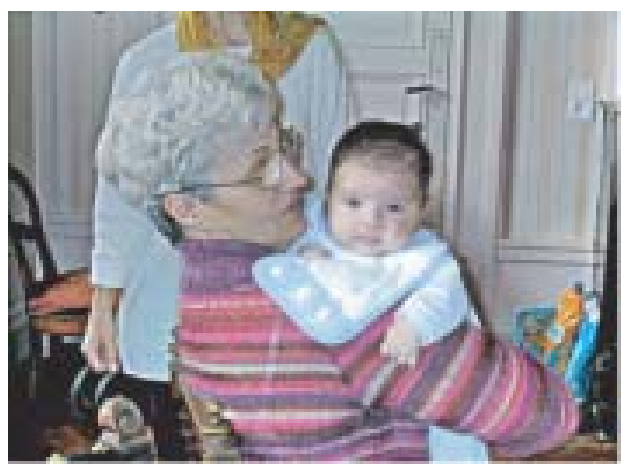

(b)

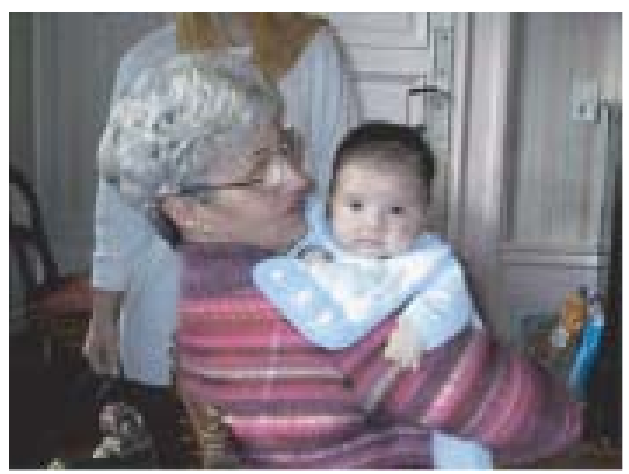

(d)

Fig. 11. (a) The original image; (b) enhanced image using multi-scale retinex theory with color restroration; (c) enhanced image using our method with $\tau=1$ and $\Delta=0.2$, followed by tone mapping using a simple polynomial tone-mapping operator; (d) enhanced image without tone mapping. Note the halo artifact in (b) around the baby's head and the lower shirt edge of the person standing behind the woman holding the baby.

and not on tone mapping. However, if required, we can apply a tone-mapping operator to our enhanced image to remove the effects of illumination and produce results akin to those produced by retinex-based methods. This is illustrated with a very simple polynomial tone-mapping operator in Figure 10.

In addition to this difference in the main principle, retinex-based methods are prone to halo artifacts, since they ignore discontinuities in illumination. This is illustrated in Figure 11. Further, these methods cause severe local or global hue shifts, as illustrated in Figure 8. Thus, a color restoration process is often required that makes the process more complex and compute-intensive. For example, the results shown in Figures 10 and 11 have been color restored, while the result in Figure 8 is not. However, note that the color-restored images in Figures 10 and 11 may look more vibrant than the images generated by our contrast-enhancement method. This is because of over saturation of colors during restoration. However, when measuring the CIELAB hue shift in Table II, we find that our method, even after tone mapping, shows a lower hue shift and, hence, preserves the hue in the original image more faithfully.

\section{SPATIALLY SELECTIVE ENHANCEMENTS}

Our method can also be applied to enhance regions of interest (ROI) in an image while maintaining a seamless boundary with the unenhanced part of the image. This can be done as a simple extension of 


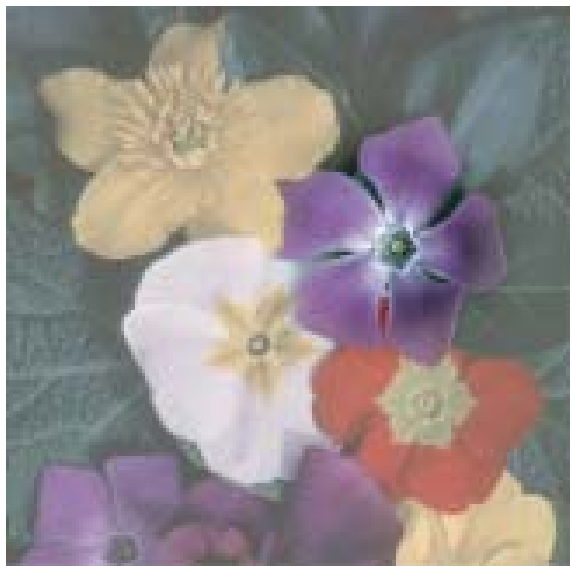

(a)

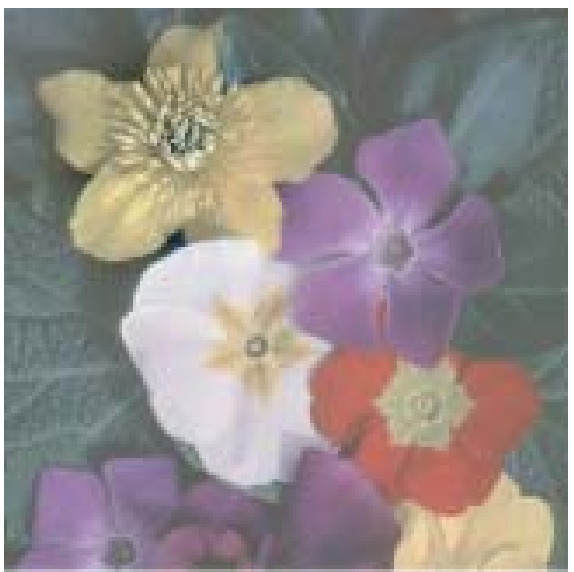

(c)

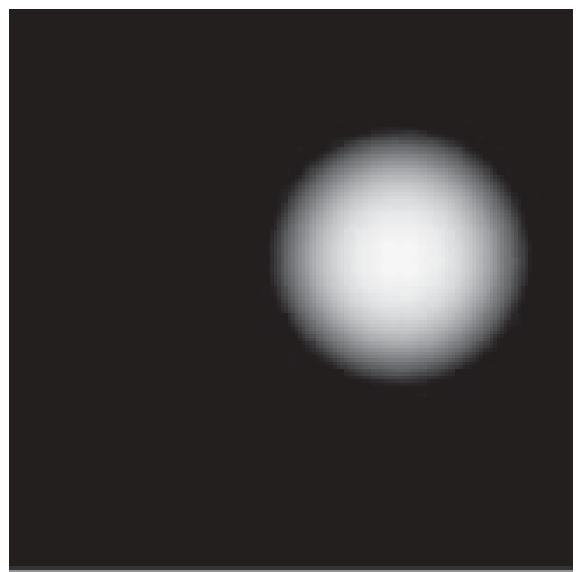

(b)

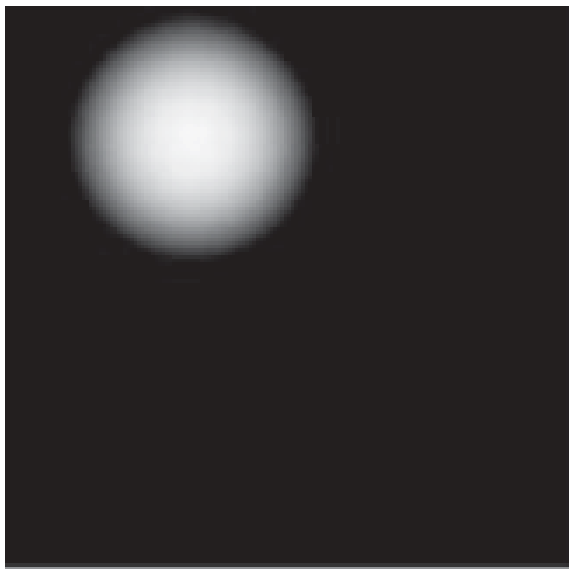

(d)

Fig. 12. (a) and (c) Seamless spatially selective contrast enhancement of the violet and yellow flower, respectively; (b) and (d) attenuation mask used to attenuate $\tau$ differently at different spatial locations to achieve the selective enhancement in (a) and (c), respectively.

the original method where the parameter $\tau$ can be varied spatially. For example, to selectively enhance a circular ROI in the image, we attenuate $\tau$ in that region spatially by a gaussian function where the peak of the gaussian occurs at the center of the circle and falls to zero beyond the radius of this circular region. Thus, the regions outside the circle do not get enhanced and the enhancement within the region falls off smoothly from the center to the fringes giving a seamless selective enhancement. Figure 12 shows the spatial mask used for attenuating $\tau$ and the result of such spatially selective enhancement for a circular region of interest. For ROIs of arbitrary shape, two approaches can be adopted. A circle enclosing the arbitrarily shaped region can be found and a Gaussian attenuation mask with appropriate radius can be applied to achieve the selective enhancements. Or, some distance-based attenuation mask can be applied that attenuates $\tau$ at any pixel proportional to the distance of the pixel from the boundary of the ROI. 


\section{CONCLUSION}

In conclusion, we use that fact that the suprathreshold human contrast sensitivity follows the Weber Law to achieve contrast enhancements of images. We apply a greedy algorithm to the image in its native resolution without requiring any expensive image segmentation operation. We pose the contrast enhancement as an optimization problem that maximizes an objective function defining the local average contrast enhancement (ACE) in an image subject to constraints that control the contrast enhancement by a single parameter $\tau$. We extend this method to color images where hue is preserved while enhancing only the luminance contrast. In addition, we vary the parameter $\tau$ spatially over the image to achieve spatially selective enhancement. Finally, we show that the ACE defined by the objective function can act as a metric to compare the contrast enhancement achieved for different methods and different parameters thereof.

Future work in this direction will include exploring the possibility of extending this to video by adding the additional temporal dimension. We also intend to pursue applications dealing with $1 \mathrm{D}$ signals, such as sound. Since our method treats the image as a height field, it could have interesting applications in terrain or mesh editing. Finally, exploring the possibility of implementing this method on graphic processing units would allow this method to be used interactively.

\section{ACKNOWLEDGMENTS}

We acknowledge Prof. Raanan Fattal of University of California, Berkeley who provided us some images for comparing and validating our results. We acknowledge NSF (Grant CCF-0514082) for partially supporting the second author of the paper.

\section{REFERENCES}

Barten, P. G. 1999. Contrast sensitivity of the human eye and its effects on image quality. SPIE - The International Society for Optical Engineering, P.O. Box 10 Bellingham Washington 98227-0010. ISBN 0-8194-3496-5.

Boccignone, G. and Picariello, A. 1997. Multiscale contrast enhancement of medical images. In Proceedings of ICASSP.

Burt, P. J. AND Adelson, E. H. 1983. A multiresolution spline with application to image mosaics. ACM Transactions on Graphics 2, 4, 217-236.

Debevec, P. E. and Malik, J. 1997. Recovering high dynamic range radiance maps from photographs. In Proceedings of ACM SIGGRAPH, 369-378.

FAtTAL, R., Lischinski, D., AND Werman, M. 2002. Gradient domain high dynamic range compression. ACM Transactions on Graphics, Proceedings of ACM Siggraph 21, 3, 249-256.

Georgeson, M. and Suldivan, G. 1975. Contrast constancy: Deblurring in human vision by spatial frequency channels. Journal of Physiology 252, 627-656.

Giongianni, E. J. And Madden, T. E. 1998. Digital Color Management: Encoding Solutions. Addison Wesley, Reading, MA.

Hanmandlu, M., JHA, D., And Sharma, R. 2000. Color image enhancement by fuzzy intensification. In Proceedings of International Conference on Pattern Recognition.

Hanmandlu, M., Jha, D., And Sharma, R. 2001. Localized contrast enhancement of color images using clustering. In Proceedings of IEEE International Conference on Information Technology: Coding and Computing (ITCC).

Kingdom, F. A. A. And Whittle, P. 1996. Contrast discrimination at high contrasts reveal the influence of local light adaptation on contrast processing. Vision Research 36, 6, 817-829.

Koenderink, J. J. $\quad$ 1984. The structure of images. Biological Cybernetics 50, 5, 363-370.

Land, E. 1964. The retinex. American Scientist 52, 2, 247-264.

Land, E. And McCann, J. 1971. Lightness and retinex theory. Journal of Optical Society of America 61, 1, 1-11.

Mantiuk, R., Myszkowski, K., AND Seidel, H.-P. S. 2006. A perceptual framework for contrast processing of high dynamic range images. ACM Transactions on Applied Perception 3, 3.

Mukhopadhyay, S. And Chanda, B. 2002. Hue preserving color image enhancement using multi-scale morphology. Indian Conference on Computer Vision, Graphics and Image Processing. 
Article $17 / 22 \quad-\quad$ A. Majumder and S. Irani

Munteanu, C. and Rosa, A. 2001. Color image enhancement using evolutionary principles and the retinex theory of color constancy. In Proceedings 2001 IEEE Signal Processing Society Workshop on Neural Networks for Signal Processing XI, 393-402.

OAKley, J. P. And Satherley, B. L. 1998. Improving image quality in poor visibility conditions using a physical model for contrast degradation. IEEE Transactions on Image Processing 7, 167-179.

PeLi, E. 1990. Contrast in complex images. Journal of Optical Society of America A 7, 10, 2032-2040.

Prez, P., Gangnet, M., and Blake, A. 2003. Poisson image editing. ACM Transactions on Graphics, Proceedings of ACM Siggraph 22, 3, 313-318.

Rahman, Z., Jobson, D. J., , AND Woodell, G. A. 1996. Multi-scale retinex for color image enhancement. IEEE International Conference on Image Processing.

Reinhard, E., Stark, M., Shirley, P., and Ferwerda, J. 2002. Photographic tone reproduction for digital images. ACM Transactions on Graphics (SIGGRAPH) 21, 3, 267-276.

Reinhard, E., Ward, G., Pattanaik, S., and Debevec, P. 2005. High Dynamic Range Imaging. Morgan Kaufmann Pub. San Francisco, CA.

Shyu, M. And LeoU, J. 1998. A geneticle algorithm approach to color image enhancement. Pattern Recognition 31, 7, 871-880.

Stark, J.-L., Murtagh, F., Candes, E. J., and Donoho, D. L. 2003. Gray and color image contrast enhancement by curvelet transform. IEEE Transactions on Image Processing 12, 6.

Tовт, A. 1990. A hierarchical morphological image decomposition. Pattern Recognition Letters 11, 4, $267-274$.

TоEт, A. 1992. Multi-scale color image enhancement. Pattern Recognition Letters 13, 3, 167-174.

Valois, R. L. D. and Valois, K. K. D. 1990. Spatial Vision. Oxford University Press, Oxford

VELDE, K. V. 1999. Multi-scale color image enhancement. In Proceedings on International Conference on Image Processing 3, $584-587$.

Whittle, P. 1986. Increments and decrements: Luminance discrimination. Vision Research 26, 10, 1677-1691.

Wilson, H. 1991. Psychophysical models of spatial vision and hyperacuity. Vision and Visual Dysfunction: Spatial Vision, D. Regan, Editor, Pan Macmillan, 64-86.

Witkin, A. P. 1983. Scale-space filtering. In Proceedings of the 7th International Joint Conference on Artificial Intelligence, $1019-1022$.

Received October 2006; revised May 2007; accepted June 2007

ACM Transactions on Applied Perception, Vol. 4, No. 3, Article 17, Publication date: July 2007. 